\title{
BONE EROSIONS AND OSTEOPHYTES IN PREMENOPAUSAL WOMEN WITH LONG-STANDING RHEUMATOID ARTHRITIS: ASSOCIATION WITH SYSTEMIC BONE INVOLVEMENT USING HR-PQCT
}

Mariana Ortega Perez ${ }^{1}$, Camille Peixoto Figueiredo ${ }^{1}$, Lucas Peixoto Sales ${ }^{1}$, Ana Cristina de Medeiros-Ribeiro ${ }^{1}$, Liliam Takayama ${ }^{1}$, Diogo Souza Domiciano ${ }^{1}$, Karina Bonfiglioli ${ }^{1}$, Valeria de Falco Caparbo ${ }^{1}$, Rosa Maria Rodrigues Pereira ${ }^{1, *}$

1. Hospital das Clinicas HCFMUSP, Faculdade de Medicina, Universidade de São Paulo, São Paulo (SP), Brazil.

*Corresponding author: rosamariarp@yahoo.com

\section{BACKGROUND}

Two patterns of bone involvement are described in systemic and localized rheumatoid arthritis (RA). Systemic bone involvement is characterized by loss of generalized bone mass, osteoporosis and risk of fractures, and the localized by periarticular osteopenia, cysts and bone erosions. High-resolution peripheral quantitative computed tomography (HR-pQCT) is an imaging methodology capable of assessing volumetric bone mineral density (VBMD) and bone microarchitecture at the distal tibia and radius, as periarticular bone lesion, catabolic (erosion) and anabolic (osteophytes) in arthropathies inflammatory. To evaluate premenopausal women with long-standing RA and to explore the relationship between parameters of systemic and localized bone involvement.

\section{METHODS}

Eighty consecutive RA premenopausal women were evaluated. Volumetric bone mineral density, microarchitecture and finite element analysis were performed using HR-pQCT at the distal radius and tibia and compared with parameters from 160 female healthy controls matched by age and body mass index. Localized bone involvement was also analyzed using HR-pQCT in the metacarpophalangeal and proximal interphalangeal joints to identify erosions and osteophytes.

\section{RESULTS}

The mean age of RA patients was $39.4 \pm 6.7$ years and disease duration $9.8 \pm 5.3$ years. Rheumatoid arthritis patients had impaired trabecular, cortical and bone strength parameters, at the distal radius and tibia, compared with healthy controls $(p<0.05)$. Bone erosions and osteophytes were found in $75 \%$ and $41.3 \%$ of patients, respectively. Comparing patients with and without erosions, at the distal radius and tibia, a lower cortical $v B M D$ (radius: $980 \pm 72$ versus $1021 \pm 47 \mathrm{mgHA} / \mathrm{cm} 3, p=0.03$; tibia: $979 \pm 47$ versus $1003 \pm 34 \mathrm{mgHA} / \mathrm{cm} 3, p=0.04$ ) and higher cortical porosity (radius: $2.8 \pm 2.5$ versus $1.8 \pm 1.6 \%, p=0.04$; and tibia: $3.7 \pm 1.6$ versus $2.7 \pm 1.6 \%, p=0.01$ ) were observed in patients with erosions. At the distal radius, osteophyte volume was positively correlated with trabecular $\operatorname{VBMD}(0.392, p=0.02)$, trabecular number $(0.381, p=0.03)$ and stiffness $(0.411, p=0.02)$, and negatively with trabecular separation $(-0.364, p=0.04)$.

\section{CONCLUSION}

This study showed that premenopausal women with long-standing RA had systemic bone fragility at peripheral sites. Moreover, erosions were associated mainly with cortical bone fragility at the distal radius and tibia, and osteophytes correlated with repair of trabecular bone at the radius.

\section{KEYWORDS}

Rheumatoid arthritis, Bone involvement, HR-pQCT, Erosion, Osteophyte. 